\title{
Herramientas para la planificación energética y ambientalmente sustentable de las áreas urbanas. Definición de Zonas Climáticas Locales en el AMM.
}

\section{Tools for energy and environmentally sustainable planning of urban areas. Definition of Local Climate Zones in MMA.}

\section{Doctorando:}

\section{María Florencia Colli}

Instituto de Ambiente, Hábitat y Energía (INAHE), Centro Científico Técnico (CCT), Consejo Nacional de Investigaciones Científicas y Técnicas de Argentina (CONICET), Mendoza - Argentina mfcolli@mendoza-conicet.gob.ar

\section{Directora}

\section{Érica N. Correa}

\section{Co-directora}

\section{Claudia F. Martinez}

\section{Resumen}

El trabajo tiene por objetivo generar una zonificación climática para el Área Metropolitana de Mendoza (AMM) que permita estandarizar el estudio del clima urbano y disponer de una base teórica sólida para la planificación y manejo de la ciudad. El modelo desarrollado es el de Zonas Climáticas Locales (LCZ) propuesto por Stewart y Oke, y para ello se ha utilizado la herramienta WUDAPT (World Urban Database Access Portal Tools).

Los resultados muestran que, las zonas climáticas identificadas dentro del área de análisis exponen correlación con el paisaje de los distintos sectores de la ciudad.

Palabras clave: clima urbano, zonificación, LCZ, morfología urbana, Área Metropolitana de Mendoza

\begin{abstract}
The work aims to generate a climatic zoning for the Mendoza Metropolitan Area (AMM) that allows to standardize the study of urban climate and to have a solid theoretical basis for planning and management of the city. The model to be developed is the Local Climate Zones (LCZ) proposed by Stewart and Oke and for this purpose it has been used as a WUDAPT (World Urban Database Access Portal Tools) tool.
\end{abstract}

The results show that, overcoming the tool limitations, the climatic zones identified within the analysis area show correlation with the landscape in different city sectors.

Keywords: urban climate, zonification, LCZ, urban morphology, AMM.

\section{Introducción}

Las ciudades impulsan cambios ambientales a nivel global y también son excepcionalmente vulnerables a las consecuencias de dicho cambio (Grimond, 2010). Además, las ciudades producen condiciones climáticas adversas, que pueden causar disconfort térmico, estrés ambiental y exposición a condiciones de contaminación e insalubridad en los habitantes urbanos (Bechtel et al, 2015). El planeamiento urbano tiene una transcendencia fundamental para 
informar, coordinar e implementar medidas que mejoren la calidad ambiental de las ciudades frente al cambio climático. En las últimas décadas la climatología urbana ha adquirido un notable desarrollo como respuesta a los problemas que acompañan al creciente proceso de urbanización, sumando nuevos puntos de vista en la ecología y en la eficiencia energética. Sin embargo, en los países en vías de desarrollo, como el nuestro, son escasas las series de datos y la información microclimática disponible.

Para estudiar el efecto combinado del clima urbano, el cambio climático en las ciudades y evaluar la vulnerabilidad de las poblaciones urbanas, se necesitan modelos climáticos avanzados. Si bien existen varios modelos aplicados (Castro Conrado et al., 2014, Fernández Garcia y Martilli, 2016, entre otros) uno de los que posee mayor aplicación es el de Zonas Climáticas Locales (LCZ) desarrollado por Stewart y Oke (2012).

El modelo de LCZ desarrollado por Stewart y Oke es un sistema de clasificación del paisaje que se basa en el clima de espacios urbanos y rurales, con el objetivo de estandarizar los estudios de UHI (Urban Heat Island/Isla de Calor Urbana). El sistema comprende la categorización de LCZ que son "regiones uniformes en cobertura del suelo, estructura, materiales y actividades humanas que se extienden entre unos cientos de metros hasta algunos kilómetros en escala horizontal". La categorización se realiza en $17 \mathrm{LCZ}, 15$ de ellas definidas por la morfología de superficie y cobertura de suelo, y 2 definidas por el uso del suelo y los materiales de construcción predominantes en cada una. El conjunto estándar es sectorizado en dos tipologías: a) construidas -LCZ 1 a 10-, y b) de cobertura de suelo - LCZ A a G. Cada una de estas zonas posee parámetros (tabla $\mathrm{N}^{\circ} 1$ ) que configuran y caracterizan las propiedades morfológicas, de cobertura superficial, propiedades radiativas y propiedades metabólicas. Cada "zona" se nombra individualmente, distinguiendo el conjunto de propiedades superficiales que las caracteriza.

Atendiendo a que los espacios urbanos de una ciudad no se ajusten a los tipos propuestos, los autores proponen como alternativa la posibilidad de realizar una subclasificación combinando tipologías. La notación resultante para las nuevas subclases es "LCZ Xai", donde:

$\mathrm{X}=$ clase superior de origen en el conjunto estándar de LCZ

$\mathrm{a}=$ es la clase primaria inferior del conjunto estándar de LCZ

i= es una variable o propiedad efímera de cobertura del suelo si corresponde

$\begin{array}{cccccc}\text { Zona Climática Local (LCZ) } & \begin{array}{c}\text { Relación } \\ \text { H/W }\end{array} & \text { SVF } & \begin{array}{c}\text { Proporción de } \\ \text { superficie del } \\ \text { suelo con la } \\ \text { cubierta del } \\ \text { edificio (\%). }\end{array} & \begin{array}{c}\text { Proporción de } \\ \text { superficie del suelo } \\ \text { con cubierta } \\ \text { impermeable (roca, } \\ \text { pavimento) (\%). }\end{array} & \begin{array}{c}\text { Construcción } \\ \text { media / altura } \\ \text { del árbol zH }\end{array} \\ \text { Antropogénico }\end{array}$

\begin{tabular}{|c|c|c|c|c|c|c|}
\hline 1-Compacto en altura & $>2$ & $0.2-0.4$ & $40-60$ & $40-60$ & $>25$ & $50-300$ \\
\hline 2- Compacto de media altura & $0.75-1.5$ & $0.3-0.6$ & $40-70$ & $30-50$ & $8-20$ & $<75$ \\
\hline 3-Compacto de baja altura & $0.75-1.5$ & $0.2-0.6$ & $40-70$ & $20-40$ & 3-8. & $<75$ \\
\hline 4-Abierto en altura. & $0.75-1.25$ & $0.5-0.7$ & $20-40$ & $30-40$ & $>25$ & $<50$ \\
\hline 5-Abierto de media altura & $0.3-075$ & $0.5-0.8$ & $20-40$ & $30-50$ & $8-20$ & $<25$ \\
\hline 6-Abierto de baja altura & $0.3-075$ & $0.6-0.9$ & $20-40$ & $20-40$ & $3-8$. & $<25$ \\
\hline 7-Construcciones bajas & $1-2$. & $0.2-0.5$ & $60-90$ & $<10$ & $2-4$ & $<35$ \\
\hline 8-Grandes Construcciones bajas & $0.1-0.3$ & $>0.7$ & $30-50$ & $40-50$ & $3-10$ & $<50$ \\
\hline 9-Construcciones dispersas & $0.1-0.25$ & $>0.8$ & $10-20$. & $<20$ & $3-8$. & $<10$ \\
\hline 10-Áreas Industriales. & $0.2-.05$ & $0.6-0.9$ & $30-30$ & $20-40$ & $5-15$ & $<300$ \\
\hline A-Bosque / Arbolado Denso & $>1$ & $<0.4$ & $<10$ & $<10$ & $<3-30$ & 0 \\
\hline B-Árboles dispersos & $0.25-0.75$ & $0.5-0.8$ & $<10$ & $<10$ & $3-15$ & 0 \\
\hline C-Arbustos & $0.25-1.0$ & $<0.9$ & $<10$ & $<10$ & $<2$ & 0 \\
\hline D-Plantas Bajas & $<0.1$ & $<0.9$ & $<10$ & $<10$ & $<1$ & 0 \\
\hline E-Rocas o pavimento & $<0.1$ & $<0.9$ & $<10$ & $<90$ & $<0.25$ & 0 \\
\hline F- Suelo descubierto o arena & $<0.1$ & $<0.9$ & $<10$ & $<10$ & $<0.25$ & 0 \\
\hline G-Agua & $<0.1$ & $<0.9$ & $<10$ & $<10$ & - & 0 \\
\hline
\end{tabular}

Tabla N¹. Parámetros superficiales para cada LCZ. Fuente Stewart et al. (2012)

Dentro de este contexto, el presente trabajo muestra los resultados alcanzados de la aplicación del modelo de Zonas Climáticas Locales propuesto por Stewart y Oke (2012) en el AMM utilizando como herramienta WUDAPT. Estos resultados responden al objetivo de tesis de "Establecer una diferenciación por zonas dentro del área metropolitana de acuerdo con la homogeneidad de un conjunto de atributos". La hipótesis considera que contar con una zonificación climática, posibilitará estandarizar el estudio del clima urbano y disponer de una base teórica sólida para su planificación. Además, permitirá identificar el potencial de cada zona para la incorporación de distintas estrategias pasivas o activas de aprovechamiento de las energías renovables, para el acondicionamiento térmico y/o la provisión de energía de los espacios interiores y exteriores de la ciudad. 


\section{Desarrollo}

El trabajo se ha desarrollado a través de las siguientes etapas:

-Análisis crítico de estado del arte, posibilidades e inconvenientes de la implementación de la metodología a nivel internacional, regional y local.

-Definición de las zonas climáticas locales en el AMM a partir de la aplicación del método WUDAPT.

\section{Análisis crítico de antecedentes}

La búsqueda bibliográfica se orientó a identificar investigaciones a nivel internacional, regional y local, que tuvieran por objeto el desarrollo y/o la aplicación de la metodología de Zonas climáticas locales (LCZ) desarrollado por Stewart y Oke (2012). Se extrajeron estudios para diferentes localizaciones geográficas y climáticas. Las decisiones de selección no han estado limitadas al tratamiento de ciudades con características similares al AMM, sino orientadas a evaluar las posibilidades de implementación del método en distintos contextos de urbanización y estructuras de ordenación del territorio. A tal fin se rastreó información en las siguientes bases de datos digitales: ScienceDirect, ProQuest, EBSCOhost, CSA Illumina, informaworld, SpringerLink, Web of Science, y Wiley Interscience. El bagaje documental obtenido fue seleccionado para conformar el universo de análisis.

\section{Definición de LCZ en el AMM a partir de la aplicación del método WUDAPT}

Se utilizó para la clasificación de las LCZ la herramienta World Urban Database y Access Portal Tools (WUDAPT). Es un proyecto comunitario de acceso libre, que busca generar un censo de ciudades de todo el mundo ofreciendo herramientas para definir las Zonas climáticas locales.

El Área Metropolitana de Mendoza, presenta severos problemas desde el punto de vista microclimático. Sus características de aridez, elevada heliofanía, escasa frecuencia e intensidad de vientos y precipitaciones; más una anomalía térmica positiva en altura y las frecuentes inversiones de temperatura, son condiciones óptimas para el fenómeno de isla de calor. Posee una intensa forestación urbana que ha sido utilizada como herramienta de acondicionamiento bioclimático de los espacios abiertos durante las horas de sol, respondiendo al modelo de "ciudad oasis". Debido a la fuerte presencia de líneas de arbolado paralelo al trasado vial es necesaria una adaptación y ajuste de la metodología propuesta internacionalmente para que su implementación sea viable en el caso de estudio.

El procedimiento metodológico se realiza a partir de los pasos especificados en el sitio web (http://www.wudapt.org/). A continuación, se explica cómo se ha desarrollado cada paso en este trabajo:

-Paso 1 Descarga de datos y software: El sitio web brinda las imágenes Landsat necesarias para comenzar con el proceso, pero sólo lo hace para un grupo de ciudades. Debido a que la ciudad de estudio no se encuentra incluida en este grupo, se procede a la selección de las imágenes satelitales Landsat 8 L1. Para evitar la influencia de la canopia o dosel forestal, que no permite la visualización por teledetección de la zona que se encuentra debajo de la misma, se seleccionan imágenes correspondientes al invierno en el hemisferio sur, día 24 de julio de 2018 a las 02:43:13 en UTC.

-Paso 2 Generación de la clasificación LCZ: Se realiza en dos etapas. En la primera, para el procesamiento y análisis de las imágenes satelitales, se utilizó el software libre QGis 3.2. Las imágenes se proyectaron en Posgar 07 Argentina Faja 2. La calibración y la corrección atmosférica de todas las bandas se realiza automáticamente mediante el método DOS1 y los niveles digitales se convierten a valores de reflectancia (Picone, 2014). En una segunda etapa, para generar las LCZ con el método WUDAPT se utiliza una Clasificación Supervisada, con las categorías de LCZ definida por Stewart y Oke (2012). Finalizada la selección de las áreas de entrenamiento, se ejecuta el algoritmo de clasificación, El resultante u output file es un archivo ráster, donde cada valor de píxel corresponde a una categoría de LCZ.

\section{Resultados}

\section{Etapa1: Análisis crítico de antecedentes}

El modelo de Zonas climáticas locales (LCZ) desarrollado por Stewart y Oke (2012), ha sido aplicado en distintos casos a nivel mundial, regional y local. A nivel mundial, Stewart (2014) realiza clasificación de LCZ utilizando observaciones de temperatura en las ciudades de Nagano, Vancouver y Uppsala. En Nagano-Japón se establecen 5 LCZ, de las cuales 4 son estándar. En Vancouver-Canadá se establecieron 8 LCZ, con 7 estándar; y en Upsala-Suecia sólo se definieron 4 LCZ, todas estándar. Chuyuan Wang y colaboradores (2018) realizan una evaluación de las LCZ en ciudades áridas de Estados Unidos de América, clasifica y evalúa LCZ para Phoenix y Las Vegas, siguiendo el método 
World Urban Database y Access Portal Tools (WUDAPT) (2017). Ambas ciudades se clasifican en zonas estándar, 7 LCZ de tipo construido y 7 LCZ de tipo de cubierta.

En Brasil Monteiro (2018) analiza la relación de LCZ con la morfología urbana, tomando como caso de estudio Campinas, San Pablo. La autora define las LCZ por la morfología de superficie, cobertura, uso de suelo y material de construcción. Se identificaron 17 Zonas Climáticas Locales en total, siendo 12 de ellas de tipologías construidas, y 5 de tipologías de cobertura de suelo. Resultaron ser en su totalidad subclases. Pezzuto y colaboradores (2013), examinan dos métodos de análisis territorial el sistema de LCZ y la Unidad de Paisaje. Se toma también el caso de estudio Campinas San Pablo-Brasil. En total se definieron 18 Unidades de Paisaje. El método consideró dos elementos de agrupación: los "Grupos" y los "tipos". Los "grupos" se caracterizan por los elementos morfológicos claves y los "tipos" morfológicos guardan características similares entre sí, percibiendo la complementariedad de métodos.

En Argentina, Piccone (2014), realiza una clasificación de la ciudad de Tandil. Se utilizaron variables físicas, características de construcción, cobertura del suelo y concentración de la población. Se reconocieron 17 LCZ. De ellas, 7 son clases puras y 10 subclases. Roca y colaboradores (2016) realizan un estudio aplicado a la ciudad de San Juan, donde se analiza la formulación y evaluación de un Modelo de Confort Urbano a microescala. Para el área metropolitana de San Juan se establecieron 8 LCZ, 7 Subclases y 1 estándar.

En Mendoza, Puliafito y colaboradores (2013) realizan un análisis de las áreas verdes y el confort térmico a microescala. Se asignaron 12 LCZ al AMM, de este total, 5 son clases estándar y 7 son subclases definidas por las particularidades que representa el arbolado urbano. Sin embargo, el trabajo no especifica cuál ha sido el método de interpolación geoespacial para definir los límites de las zonas climáticas y su nivel de ajuste; y aunque hace referencia a datos de temperatura del aire, no hay contrastación térmica entre tipos de zonas semejantes, pero con distinta localización dentro del AMM. En este sentido, se hace referencia a la toma de datos de temperatura, pero los datos meteorológicos son mediciones 2003-2005, en tanto que los resultados obtenidos de la caracterización morfológica y tecnológica corresponden a 2013. De acuerdo a la metodología LCZ, que establece que las mediciones sean simultáneas y temporalmente coincidentes con la caracterización morfológica de los puntos tomados como referencia; resulta importante evitar la disociación temporal entre la toma de datos meteorológicos y la caracterización de la trama urbana para generar una correcta zonificación. Es necesario tener en cuenta, que el AMM reporta en los últimos 10 años un proceso de trasformación donde las zonas periurbanas o de transición son dinámicas y las variables microclimáticas también han evidenciado modificaciones (Sosa, et al., 2016).

En términos generales, la limitación fundamental del modelo es que no permite capturar las peculiaridades de cada sitio analizado, ya que es un sistema reduccionista, esto deriva en que su capacidad de descripción, análisis y explicación tenga ciertas restricciones. Para los casos en que una ciudad no se ajuste a los tipos propuestos, Stewart y Oke (2012) proponen la realización de una subclasificación combinando tipologías. Las subclases están justificadas cuando las características secundarias del sitio afectan el clima local o cuyas características pueden estar relacionadas con los objetivos particulares de una investigación climática. Se dan advertencias para su uso: - Primero, el sistema propuesto no proporciona valores de propiedad para subclases. -En segundo lugar, no se espera que las propiedades de una subclase LCZ difieran significativamente de las propiedades de cualquiera de sus clases de origen. -Tercero, el propósito de las LCZ es facilitar el proceso de clasificación de sitios y reportes de datos para las investigaciones de UHI, crear demasiadas subclases socava esta función principal. De modo específico, la configuración urbana del AMM, en lo que refiere a la fuerte presencia de arbolado en alineación de calles, característica que no tiene la misma expresión en los países del hemisferio Norte, dónde la metodología fue concebida, demanda una adaptación y un ajuste a la metodología propuesta internacionalmente para que su implementación sea viable en la región.

Todo lo discutido previamente, pone en evidencia la necesidad de analizar con mayor profundidad la definición de las zonas climáticas locales, para la definición de las LCZ en el AMM.

\section{Etapa 2: Definición de LCZ en el AMM a partir de la aplicación del método WUDAPT}

Aplicado el método WUDAPT en el área de estudio el output file de la aplicación es un mapa (Figura $\mathrm{N}^{\circ} 3$ ) donde se representan las LCZ del AMM y su entorno. En el mapa se puede observar la zona urbana y periurbana del AMM, donde la Zona climática Local que predomina es "LCZ-6 Abierto de baja altura" alcanzando un porcentaje del 27,55\%, localizada fundamentalmente en la zona central de la mancha urbana, con edificios separados de baja altura ( 1 a 3 pisos). La LCZ-8 "Grandes construcciones bajas" sigue en porcentaje con un 22,71\%. Esta zona se caracteriza por poseer grandes edificaciones bajas y separadas entre sí. Hacia el este, esta zona posee heterogeneidad de usos, industrial y de depósito, salpicado con barrios cerrados cuya expansión ha crecido en la última década (Unicipio, 2018). En tercer lugar, en orden decreciente (21,42\%) se encuentra la categoría LCZ-3 "Compacto de baja altura", localizándose en la zona central de la ciudad de Mendoza, caracterizada por ser el centro administrativo, financiero y comercial de la provincia, con edificación densa de baja altura (1 a 3 pisos). Esta clase se encuentra también en 
cabeceras departamentales y en sus entornos inmediatos. Siguen en porcentaje LCZ-9 "Construcciones dispersas" (14,87\%), LCZ-5 "Abierto de mediana altura" (8,87\%) y LCZ-2 "Compacto de media altura" (4,58\%). Al oeste se observa un importante sector con las clases A y B, que representa al Parque General San Martín (374 has.). Al norte y este de la mancha urbana, predomina la zona D-plantas bajas, que se corresponde con el cinturón productivo del Oasis norte, representado por un paisaje dominado por cultivos frutihortícolas. El sector del piedemonte está categorizado con las clases E-Roca y F-Suelo desnudo, encontrando manchas dispersas de vegetación autóctona.

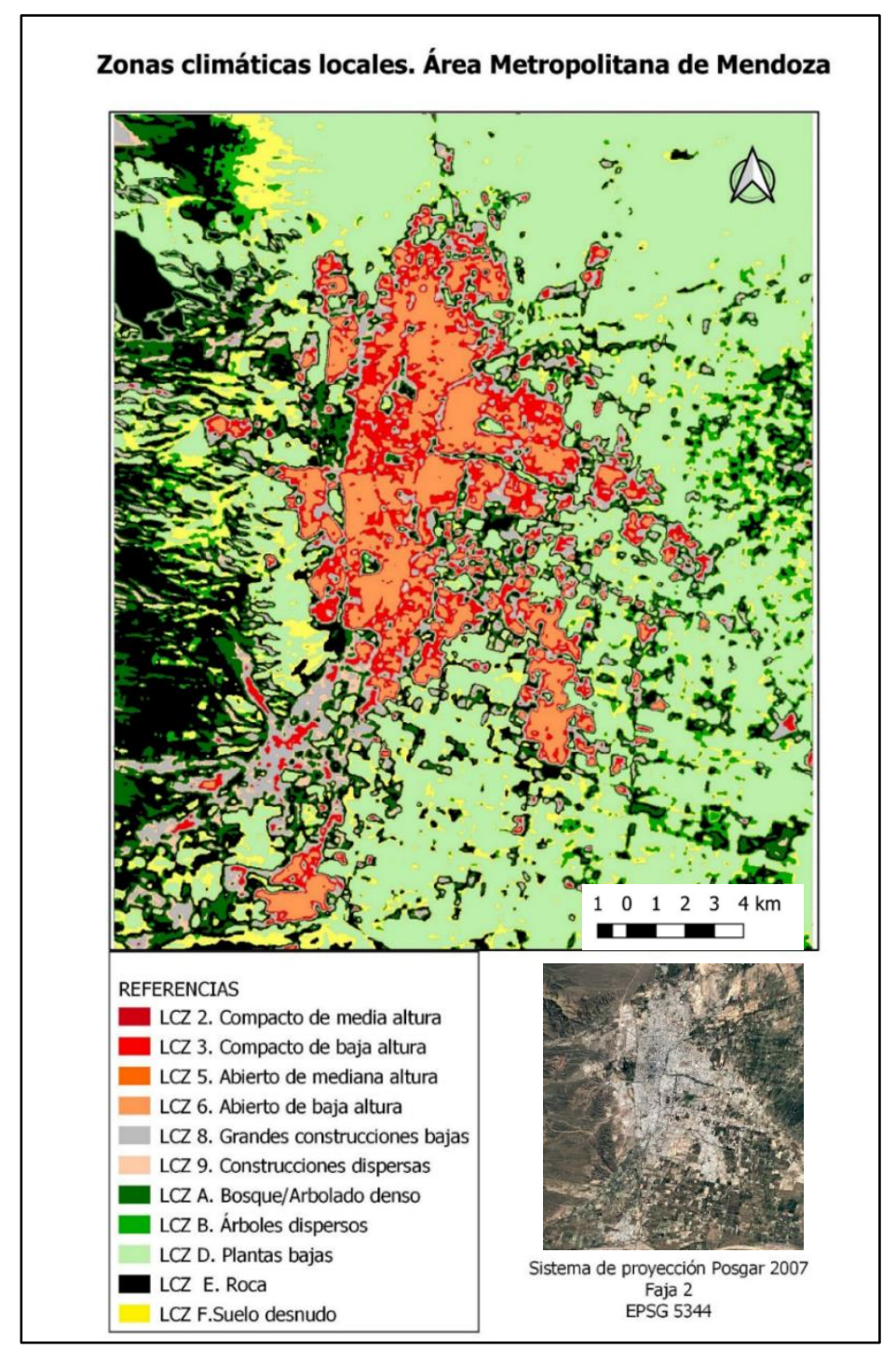

\section{Conclusiones}

Figura 2. Zonas Climáticas Locales del AMM. Fuente: Elaboración de las autoras.

El sistema de LCZ proporciona una discretización simple e integral del paisaje urbano, permitiendo estandarizar los estudios del clima urbano en general. Tiene como objetivo lograr un equilibrio entre la exactitud y la aplicabilidad, ya que se considera como un punto de partida óptimo para la recolección de los datos, la definición de la geometría urbana y las características microclimáticas de las distintas zonas. La aplicación integral de la metodología permite alcanzar el objetivo principal propuesto por sus autores que es estandarizar los estudios de clima urbano y la UHI.

Se recurre al método WUDAPT para realizar una clasificación de las LCZ. Con el desarrollo de esta técnica para la estación invernal para evitar la influencia del dosel forestal, se ha logrado como resultado la definición de las zonas climáticas para el AMM. Se definieron 11 clases, 6 de tipología de edificación y 5 de cobertura. Con respecto a las zonas definidas por tipología de construcción la LCZ-6 "Abierto de baja altura" es la que posee mayor superficie, mientras que la LCZ -2 "Compacto de media altura" la menor en tamaño. Fuera de la mancha urbana se registran las LCZ de cobertura, fundamentalmente de roca al oeste y cubierta vegetal de baja altura al este.

Analizando la herramienta WUDAPT se concluye que, si bien es un proceso con numerosos pasos y múltiples variables a considerar, se ejecuta de manera sencilla y económica ya que, teniendo datos y conocimientos del área de 
estudio, se puede realizar en su totalidad mediante teledetección. Es importante verificar que las zonas hayan sido correctamente clasificadas, debido a que, en el proceso de clasificación automática pueden confundirse clases que posean firmas espectrales similares. Es decir que, si bien es una herramienta muy útil, es una primera aproximación a la clasificación. Resulta fundamental complementar el método con la corroboración o relevamiento a campo y el ajuste mediante los parámetros definidos por Stewart y Oke. Actualmente se está trabajando sobre la etapa de verificación de parámetros morfológicos y microclimáticos de la zona de estudio.

\section{Referencias}

Bechtel, B.; Paul J.; Böhner, J.; Ching, J.; Conrad, O.; Feddema, J.; Mills, G.; See, L.; Iain, S. (2015). Mapping Local Climate Zones for a Worldwide Database of the Form and Function of Cities. International Journal of Geographic Information, 4, 199-219. February 2015International Journal of Geo-Information 4(1):199-219. DOI: 10.3390/ijgi4010199.

Castro Conrado, Y.; Fernández Figueroa, E.; Álvarez, A, López, A. (2014). Morfología urbana en la ciudad de Sagua la Grande. Arquitectura y Urbanismo. Vol.35 (3), 50-68.

Fernández García, F.; Martilli, A. (2016). En: Estudio de detalle del Clima Urbano De Madrid. Último acceso: 20 de febrero, 2020.

https://www.madrid.es/UnidadesDescentralizadas/Sostenibilidad/EspeInf/EnergiayCC/04CambioClimatico/4cEstuClim aUrb/Ficheros/EstuClimaUrbaMadWeb2016.pdf.

EarthExplorer. United States Geological Survey. https://earthexplorer.usgs.gov/ .

Grimmond C.S.B.; Roth M.; Oke T.R.; Y.C. Au; Best M.; R. Betts; Carmichael G.; Cleugh H.; Dabberdt W.; Emmanuel R.; Freita E.; Fortuniak K.; Hannal S.; Klein P.; Kalkstein L.S.; C.H. Liu; Nickson A.; Pearlmutter D.; Sailor D. J; Voogt. (2010) Climate and More Sustainable Cities: Climate Information for Improved Planning and Management of Cities. Procedia Environmental Sciences 1 (2010) 247-274.

Monteiro, V. (2018). Zonas Climáticas Locais E A Relação Com A Morfologia Urbana. Estudo De Caso: Campinas/Sp. En: Biblioteca Digital Pontificia Universidade de Campinas. Último acceso: 13 de Diciembre, 2019, de http://tede.bibliotecadigital.puc-campinas.edu.br:8080/jspui/handle/tede/1047?mode=full.

Pezzuto, C.; De La Silva, J. M. P. (2013). Métodos de Análisis del Recorte Territorial por medio de la Zona Climática Local y Unidad de Paisaje: Estudio de Caso en el Municipio de Campinas. En: XII ENCAC. Brasilia, Brasil. 25-27 Setembro. Artículo.

Piccone, N. (2014). En: Researchgate.net. Último acceso: 20 de Febrero 2020 de:

https://www.researchgate.net/profile/Natasha_Picone/publication/301495626_Clima_Urbano_de_la_ciudad_de_Tandil/li nks/5716788308aec49c999cbddc/Clima-Urbano-de-la-ciudad-de-Tandil.pdf. June 2014 DOI:

10.13140/RG.2.1.2083.8808.

Puliafito, S.; Bochaca, F.; Allende, D.; Fernández, R. (2013). Green áreas and microscale thermal comfort in arid environments: A case study in Mendoza, Argentina. Atmospheric and Climate Sciences, 3, 372-384. Atmospheric and Climate Sciences · January 2013 DOI: 10.4236/acs.2013.

Roca, G.; Puliafito, S.; Allende, D.; Ruggieri, F.; Pascual, R. (2016). Modelado urbano a microescala: contribución al confort urbano de ecosistemas áridos. Revista AVERMA, 4, 01.77-01.88.

Sosa, M.B.; Correa Cantaloube, E.;Cantón, M.A. (2016). ¿En qué medida la forma urbana afecta al comportamiento térmico exterior? Un estudio para reducir la isla de calor urbana en una ciudad árida. En: I ENCACS 2016. La Plata, Buenos Aires, Argentina. 23-27 Mayo. pp. 91-102.

Stewart, I. D.; Oke, T. R.; Krayenhoff, E. S. (2014). Evaluation Of The Local Climate Zone Scheme Using Temperature Observations And Model Simulations. International Journal of Climatology, 34.1062 - 1080. DOI: 10.1002/joc.3746.

Stewart, I. D.; Oke, T. R. (2012). Local climate zones for urban temperature studies. Bulletin of the American Meteorological Society, 93 (12), 1879-1900. DOI: 10.1175/BAMS-D-11-00019.1.

Unicipio, (2018). Secretaría de Ambiente y Ordenamiento Territorial. Último acceso: 19 de Febrero, 2020, de http://www.unicipio.mendoza.gov.ar/.

Wang, C.; Ariane, M.; Myint, S.; Kapla, S.; Brazel, A.J., Lukasczyk, J. (2018). Assessing local climate zones in arid cities: The case of Phoenix, Arizona and Las Vegas, Nevada. Journal of Photogram. and Remote Sensing, 141, 59-71.

World Urban Database y Access Portal Tools, (2020). Último acceso: 19 de Febrero, 2020 de http://www.wudapt.org/ . 